\title{
Promoting Healthy Family Behaviors in the Primary Care Setting
}

Katrina Weirauch, DO | Julie Phillips, MD, MPH

Published: 2/8/2017 | DOI: 10.22454/PRiMER.2017.1.2

\section{Abstract}

Introduction: Pediatric obesity is an increasingly prevalent problem. Several studies have examined prevention and treatment strategies. The majority of effective studies involved school or community interventions. With health care becoming more collaborative, we hypothesized that a behavioral health specialist may be effective in executing multifaceted interventions with families of at-risk patients.

Methods: This is a prospective randomized study, evaluating impact of intervention with a behavioral specialist on lifestyle risk factors for pediatric obesity in children. At-risk behaviors were identified with a screening tool from the Healthy Kids, Healthy Michigan Clinical Decision Tools, based on the 2007 American Academy of Pediatrics guidelines on pediatric obesity. An intervention group received ongoing care from the behavioral specialist over three months, including motivational interviewing and cognitive behavioral therapy. Participants were compared with a control group receiving usual care.

Results: There was no significant difference between the intervention and control group regarding change in number of risk factors. However, both groups had a reduced number of risk factors at follow-up. The control group had a significant change in number of risk factors after the intervention.

Conclusion: There was no statistically significant difference between the two groups. However, it is notable that both groups saw significant decreases in total number of risk factors. The only addition to usual care provided to the control group was use of the screening tool. Our results indicate that the use of a screening tool and brief physician intervention may be an effective means for improving healthy behaviors within families.

\section{Introduction}

According to the Center for Disease Control, obesity has doubled in children and quadrupled in adolescents over the past 30 years. ${ }^{1}$ This change has implications for both the immediate health of children and future health outcomes. Obese children are at greater risk for cardiovascular disease, diabetes, sleep apnea, joint disease, and psychological complications. ${ }^{1,2}$ They are also more likely to have these complications in adulthood. ${ }^{3}$ Obesity is challenging to reverse once established and tends to continue into adulthood. ${ }^{4}$

Multifaceted approaches appear to be more effective than targeting single risk factors in preventing and treating obesity. ${ }^{5}$ Primary care providers are in a unique position to educate families on behavioral risk factors and help prevent pediatric obesity. However, busy primary care physicians often find it difficult to implement multifaceted interventions within the constraints of limited appointment times and competing priorities. In one survey, pediatricians described a lack of support services, lack of motivation, and lack of parent involvement as barriers to physician-led intervention. ${ }^{6}$ However, in a patientcentered medical home model, ${ }^{7}$ collaborative teams may help overcome these barriers. Several social work studies have been done regarding individual and systemic risk factors for pediatric obesity and many general recommendations are 
proposed. ${ }^{8,9,10,11}$ However, very little data exist regarding efficacy of primary care based interventions. ${ }^{8}$ Specifically, the use of a LMSW in the primary care setting has not yet been studied as a way to decrease risk factors for pediatric obesity. ${ }^{10}$ We hypothesized that utilizing a pediatric licensed medical social worker (LMSW) may be an effective way to execute multifaceted interventions in a primary care setting with families of pediatric patients who are overweight, obese, or at risk for obesity.

\section{Methods}

This prospective randomized study examined the effect of LMSW-led interventions on behaviors of children who were overweight, obese, or had risk factors for obesity. The study was conducted in an urban family medicine residency clinic in the Midwestern United States and approved by the Michigan State University Institutional Review Board.

Children and their families were recruited from July 2015 to September 2015. Participants were drawn from the patient panels of six resident physicians. Participants were added on a rolling basis, using a modified stepped-wedge design. Over the course of 1 month, each resident's well child exam patients were placed in the control group; during a subsequent month, the resident's patients were placed in the intervention group. The timing of each resident's enrollment was randomized.

Children and their families were enrolled at their yearly well-child office visits. Participants were eligible if they were between the ages of 2 and 17 and had BMI greater than the $85^{\text {th }}$ percentile or a behavior that placed them at risk for obesity.

Demographic information is found in Table 1. At-risk behaviors were identified with a clinical decision tool (http://www.michigan.gov/documents/mdch/CDSTools_3-18-11_351358_7.pdf (http://www.michigan.gov/documents /mdch/CDSTools_3-18-11_351358_7.pdf)), based on the 2007 American Academy of Pediatrics guidelines, ${ }^{12}$ which was designed by the Healthy Kids, Healthy Michigan initiative. ${ }^{13}$ Written consent was obtained from parents and assent was obtained from children.

The control group received usual care following use of the screening tool. This typically involved a brief intervention from the primary care resident provider, who had access to the screening tool, during the well child appointment. In addition to provider-initiated interventions, the intervention group participated in ongoing support from a LMSW over the following three months. This support included in-person visits, phone calls, or both. The LMSW used any interventions or tools she deemed most beneficial to the family. In this way, the intervention was the use of the LMSW, rather than any specific tool. The most commonly utilized techniques were motivational interviewing and cognitive behavioral therapy, while the most heavily employed tools were taken from MyPlate.gov. ${ }^{14}$ Both groups were reassessed three months after their enrollment using the same screening tool to assess change in behavior.

Chi-square analyses and t-tests were used to compare differences in demographic characteristics between the intervention and control groups. Because there was a significant difference in the ages of the two groups [Table 1], intervention and control groups were compared using a one-way between-group analysis of co-variance (ANCOVA) with age and change in number of risk factors as the independent variables.

\section{Results}

Fifty-six children enrolled in the study; of these, $41(73 \%)$ completed all portions of the study and were included in the analysis. Of these, 13 were in the intervention group and 28 were in the control group. There were no significant differences between the intervention and control groups in race/ethnicity, gender, and number of risk factors. However, as described above, there was a difference in the ages between the two groups. In ANCOVA, there was no significant difference between the intervention and control group in the change in number of risk factors. (The mean number of risk factors after intervention was 1.3 in the intervention group, and 1.4 in the control group; $P=0.599)$. Because both the control group and the intervention group had fewer risk factors after the study, a paired samples t-test was conducted to determine whether the control group's improvement was statistically significant. The control group did have a significant change in the number of risk factors before and after the intervention (mean of 3.3 risk factors before the intervention, 1.4 after; $P<0.000$ ). 
Finally, we analyzed each risk factor individually to see which risk factors improved in the control group after the intervention. There was an improvement by at least one of the participants in every risk factor, with four individual risk factors reaching statistical significance (Table 2), (Figure 1).

\section{Conclusion}

This social work intervention did not appear to be effective in changing behavior in this short term intervention. However, more studies are needed to assess use of LMSWs, advanced practitioners, and other staff in preventing pediatric obesity prevention in primary care.

This study has several important limitations. The small sample size may have caused small effects to be missed. We also did not reallocate participants after randomization to create balanced demographics between intervention and control groups. As a result, there was a significant age difference between the two groups. Furthermore, the control group had many more participants than the intervention group. This design increased the likelihood of clustering (by both physician and family). Additionally, the participant ages ranged from 2 to 17. It is possible that our intervention may have had different levels of effectiveness for children of different ages, but our study design would have missed this outcome. Our study also used only one social worker, and therefore the outcome may not be generalizable. A study with more than one LMSW, perhaps with different education or experience, may show a beneficial outcome that we were not able to demonstrate. Finally, the outcome may have been affected by a short follow up period and responder bias. Additional studies should be performed on a larger scale, using multiple clinicians, and across multiple institutions to better evaluate the hypotheses. Patients could also be followed over a longer period of time to evaluate sustained behavior change and change in BMI percentile.

While there was no significant difference between the control and intervention groups, both groups had fewer risk factors at the follow-up assessment. Our results indicate that the simple use of a health screening tool and brief physician intervention may be a very effective means for promoting healthy behaviors. Completing the tool may have prompted patients and parents to more honestly assess their behaviors. The screening tool may have also changed the discussion between the patient (or parent/guardian) and physician. Perhaps physicians used it as a focus for risk factor discussion. Use of the screening tool may have prompted physicians to discuss obesity risks with patients and parents with normal BMIs. Regardless, primary care offices may find the screening tool to be beneficial in encouraging behavior changes. This study shows that use of the tool as a locus for patient and parent education by physicians may prevent pediatric obesity. Use of this screening tool should be studied more broadly. 


\section{Tables and Figures}

Table 1: Demographics of Participants

\begin{tabular}{|c|c|c|c|}
\hline & Control & Intervention & Total \\
\hline \multicolumn{4}{|l|}{ Gender } \\
\hline Female & 12 & 7 & 19 \\
\hline Male & 16 & 6 & 22 \\
\hline \multicolumn{4}{|l|}{ Ethnicity } \\
\hline African-American & 12 & 9 & 21 \\
\hline Asian & 0 & 0 & 0 \\
\hline Caucasian & 6 & 2 & 8 \\
\hline Latino & 6 & 0 & 6 \\
\hline Other/Combination & 4 & 2 & 6 \\
\hline \multicolumn{4}{|l|}{ Age (years) ${ }^{\star}$} \\
\hline $2-4$ & 7 & 0 & 7 \\
\hline $5-7$ & 5 & 2 & 7 \\
\hline $8-10$ & 7 & 4 & 11 \\
\hline $11-13$ & 6 & 3 & 9 \\
\hline $14-17$ & 3 & 4 & 7 \\
\hline Total & 28 & 13 & 41 \\
\hline
\end{tabular}

*Statistically significant difference between control and intervention groups, $\mathrm{P}<0.05$

Table 2: Number of Obesity Risk Factors Before and After a Pediatric Obesity Intervention

\begin{tabular}{|l|c|c|c|c|c|}
\hline & Number of Participants & Mean & Median & Range & Standard Deviation \\
\hline Before Intervention & \multicolumn{5}{|l|}{} \\
\hline Intervention Group & 13 & 3.2 & 3.5 & $0-6$ & 1.8 \\
\hline Control Group & 28 & 3.3 & 4 & 0.7 & 1.9 \\
\hline After Intervention & 13 & 1.3 & 1 & $0-4$ & 1.2 \\
\hline Intervention Group & 28 & 1.4 & 0 & $0-4$ & 1.2 \\
\hline Control Group & \multicolumn{7}{|l|}{} \\
\hline
\end{tabular}

No significant difference between groups $(P=0.599)$. 
Figure 1: Prevalence of Individual Risk Factors Before and After Intervention

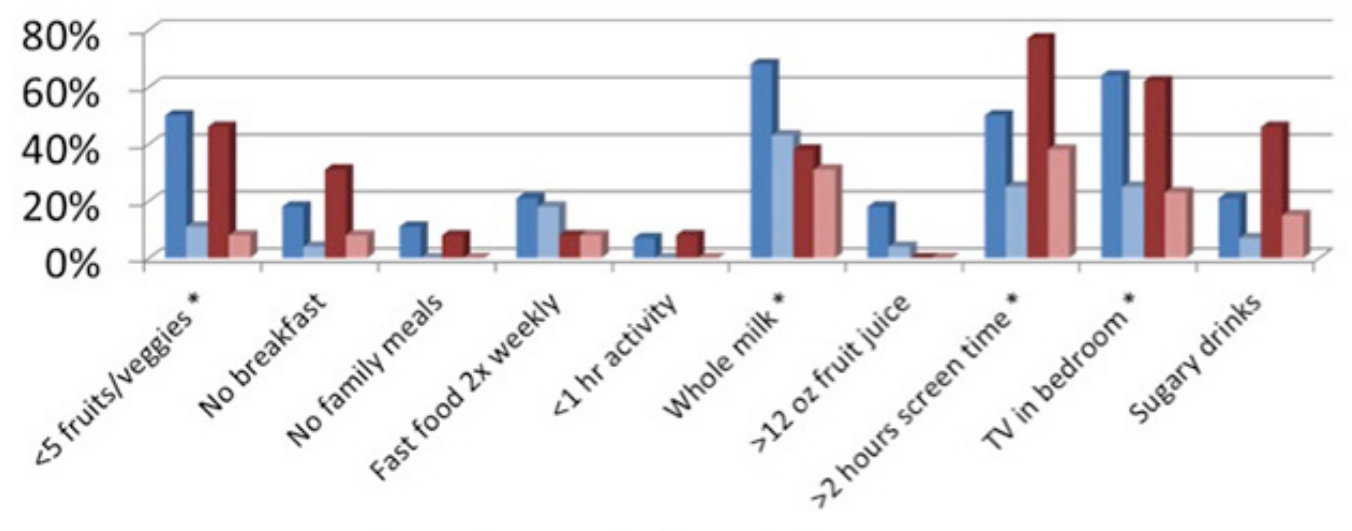

= \% of Control Group with risk factor before intervention

= \% of Control Group with risk factor after intervention

m \% of Intervention Group with risk factor before intervention

= \% of Intervention Group with risk factor after intervention

-Statistically significant change in control group, $p<0.05$

\section{Acknowledgements}

The researchers thank Allison Vinton, LMSW, for her significant time and effort dedicated to patient and family counseling to support this study.

\section{Corresponding Author}

Katrina Weirauch, DO

Michigan State University, Sparrow Family Medicine Residency Program. 1651 W Lake Lansing Rd \#300, East Lansing, MI 48823. Ph: (517)354-8686.

Katrinawe14@gmail.com (mailto:Katrinawe14@gmail.com)

\section{Author Affiliations}

Katrina Weirauch, DO - Michigan State University, Sparrow Family Medicine Residency Program

Julie Phillips, MD, MPH - Michigan State University, Sparrow Family Medicine Residency Program

\section{References}

1. Healthy Schools, Data and Statistics. (2015). Retrieved from www.cdc.gov

2. Seburg, EM, Olson-Bullis, BA, Bredeson, DM, Hayes, MG, Sherwood, NE. (2015). A Review of Primary Care-Based Childhood Obesity Prevention and Treatment Interventions. Current Obesity Reports, 4(2), 157-173. doi:10.1007/s13679-015-0160

3. Benjamin, R. M. (2010). The Surgeon General's vision for a healthy and fit nation. S. Department of Health and Human Services., 125, 514-515. https://doi.org/D - NLM: PMC2821841 EDAT- 2010/03/20 06:00 MHDA- 2010/04/03 06:00 CRDT2010/03/20 06:00 PST - ppublish

4. Whitaker, R. C., Wright, J. A., Pepe, M. S., Seidel, K. D., \& Dietz, W. H. (1997). Predicting Obesity in Young Adulthood from Childhood and Parental Obesity. New England Journal of Medicine, 337(13), 869-873. doi:10.1056/NEJM199709253371301

5. Waters E, de Silva-Sanigorski A, Hall BJ, Brown, T, Campbell, KJ, Gao Y, Summerbell, CD. (2011). Interventions for preventing obesity in children. The Cochrane Database of Systematic Reviews, (12), CD001871. doi:10.1002/14651858.CD001871.pub3. www.cochranelibrary.com 
6. Story MT, Neumark-Stzainer DR, Sherwood NE, Holt K, Sofka D, Trowbridge FL, Barlow SE. Management of child and adolescent obesity: attitudes, barriers, skills, and training needs among healthcare professionals. Pediatrics, 110(1): 210-4.

7. Akinci F, Patel PM. Quality improvement in healthcare delivery utilizing the patient-centered medical home model. Hosp Top, 92(4), 96-104. doi:10.1080/00185868.2014.968493.

8. Lawrence S, Hazlett R, Hightower P. Understanding and acting on the growing childhood and adolescent weight crisis: a role for social work. Health \& Social Work, 35(2), 147-153.

9. Juby C, Meyer, EE. Child nutrition policies and recommendations. Journal of Social Work, 11(4), 375-386. doi:10.1177/1468017310379451

10. Melius, J. Exploring social workers' use of the socioecological model as an intervention and research framework for treating overweight or obese clients. Social Work (United States), 60(1), 55-63. doi:10.1093/sw/swu048

11. Melius, J. (2013). Overweight and Obesity in Minority Children and Implications for Family and Community Social Work. Social Work in Public Health, 28(October), 119-128. doi:10.1080/19371918.2011.560821

12. Barlow S, Committee E. Expert Committee Recommendations Regarding the Prevention, Assessment, and Treatment of Child and Adolescent Overweight and Obesity: Summary Report. Pediatrics, 120(suppl 4), S164--S192. doi:10.1542/peds.2007-2329C

13. Advocates of Healthy Weight in Children (2011). Retrieved from http://www.michigan.gov/documents /mdch/CDSTools_3-18-11_351358_7.pdf.

14. United States Department of Agriculture (2016). MyPlate. Retreieved from ChooseMyPlate.gov.

Copyright () 2017 by the Society of Teachers of Family Medicine 